Nloman 2019, 37(2), 75-81

Revista de Psicologia, Ciències de l'Eduació i de l'Esport

ISSN: $1138-3194$

CFacultat de Psicologia, Ciències de l'Educació i de l'Esport Blanquerna

Universitat Ramon Llull

cc)(\$)

BY NC SA

\title{
Comparativa del uso educativo de las redes sociales en los grados de Maestro: universidades presenciales y online
}

\author{
Raquel Gil-Fernández ${ }^{1}$, Diego Calderón-Garrido ${ }^{1}$, Alicia León-Gómez ${ }^{1}$ \\ \& Carolina Martin-Piñol ${ }^{2}$ \\ ${ }^{1}$ Universidad Internacional de La Rioja \\ 2Universidad de Barcelona \\ Recibido: 2019-3-15 \\ Aceptado: 2019-6-5
}

Comparativa del uso educativo de las redes sociales en los grados de Maestro: universidades presenciales y online

Resumen. El uso de las redes sociales por parte de los maestros en su formación inicial es uno de los indicadores de su competencia digital. En este trabajo se analizan el uso diario y el uso educativo de las redes sociales genéricas entre los estudiantes de Grado de Maestro comparando los datos obtenidos en una universidad online con los de varias universidades presenciales. Para alcanzar los objetivos, se diseñó un cuestionario, obteniendo una muestra validada de 340 estudiantes. Los resultados revelaron que las redes sociales más usadas son WhatsApp, YouTube y Facebook. En cuanto a su uso educativo, se ha comprobado que se debería implementar. Los resultados mostraron diferencias en el uso educativo que se les da a las distintas redes entre las universidades que operan en un entorno presencial y aquellas que operan en uno virtual.

Palabras clave: competencia digital docente; redes sociales; educación virtual; innovación docente mediante uso de las TIC; alfabetización digital en educación

A comparison of the educational use of social networks in teacher training degrees: faceto-face versus online universities.

\begin{abstract}
Summary. The use of social networks by teachers in their initial training is one of the indicators of their digital competence. In this paper, the daily use and educational use of generic social networks among Master's Degree students is analyzed by comparing the data obtained at an online university with those of several face-to-face universities. To achieve the objectives, a questionnaire was designed, and a validated sample of 340 students was recruited. The results revealed that the most commonly used social networks are WhatsApp, YouTube and Facebook. Regarding their educational use, it has been proven that it should be implemented. The results showed differences between the educational use of the different networks in the universities that operate in a face-to-face environment and the uses by the students at the online university.
\end{abstract}

Keywords: teaching digital competence; social networks; virtual education; teaching innovation through the use of ICT; digital literacy in education

Correspondence

Raquel Gil Fernández

Universidad Internacional de La Rioja

ORCID: 0000-0001-9881-8641

raquel.gilfernandez@unir.net 


\section{Introducción}

La legislación española considera la competencia digital una de las prioridades formativas en todo el alumnado (Orden ECD/65/2015, Recomendación del Parlamento Europeo 2006/962/CE). Según este planteamiento, se han realizado numerosas investigaciones en distintos niveles educativos (Callaghan \& Bower, 2012; Fondevila, Olmo \& Sierra, 2012; Haro, 2011; López-Fernández, 2016; Marina, Pellicer \& Manos, 2015; Sloep \& Berlanga, 2011; Tirado \& Martínez, 2010). Asimismo, el Espacio Europeo de Educación Superior deja clara la existencia de una demanda en relación con el perfil docente, insistiendo en la adquisición de una competencia digital que permita a los profesionales asumir los desafíos necesarios para adquirir un pensamiento reflexivo y tener la capacidad de implementar metodologías activas (Serrat, 2015), de manera que los discentes alcancen una formación global que permita su correcta inserción laboral. Es decir, se trata de que un docente sea competente en términos digitales para poder desarrollar dicha competencia en el discente. $\mathrm{O}$, en el caso que nos ocupa, que un futuro maestro sea competente en dichos términos digitales para poder desarrollar dicha competencia en su futuro alumnado.

En esta investigación se ha partido de la premisa de considerar que un indicador relevante de la adquisición de la citada competencia digital es el uso educativo que se les da a las redes sociales genéricas más comunes. Esta idea une el mundo de fuera del aula con el de dentro del aula, lo cual es necesario en el ámbito de una sociedad digitalizada para poder plantear un correcto desempeño del proceso enseñanza-aprendizaje (Calderón, Forés \& Gustems, 2015). Así pues, y tal como apuntaban García y Martín (2013) respecto a los docentes $y$, por ende, a los futuros maestros, no se trata de abordar la enseñanza del uso de las redes sociales, sino de enfocar su trabajo como medio de difusión, tratamiento y profundización de los contenidos que se abordan en el aula. Tal y como plantean Túñez y Sixto (2012), se trata simplemente de pivotar sobre un claro axioma: en vez de motivar al alumno a entrar en los entornos educativos, ¿por qué no llevar los contenidos académicos a los entornos en los que acostumbra a estar el alumno? Es evidente que si los alumnos emplean las redes sociales fuera de un entorno educativo, estas se podrían explotar para potenciar el binomio enseñanza-aprendizaje.

En este trabajo, siguiendo a López-Frías (2014), se ha entendido por "redes sociales genéricas" aquellas estructuras de interrelación en las que se produce un intercambio social, pues el interés ha sido conocer el uso de estas y no el de las redes sociales enfocadas a un uso específicamente profesional o científico, si bien, siguiendo a la misma autora, asumimos que el uso de estas últimas no es excluyente, ya que de hecho puede - y suele - ser complementario. La falta de concreción de las redes genéricas permite darles un uso muy flexible, diverso e innovador que se adapta a los objetivos de cada usuario (González \& Muñoz, 2016).
Sin embargo, no se puede olvidar que hay trabajos que detectan que el uso de redes sociales con fines educativos no se está implementando con la intensidad que sería deseable a pesar de sus beneficios (Altuzarra, Gálvez \& Gónzalez, 2018). Esto es debido a que los alumnos en ocasiones no disponen de experiencias reales que les valgan de ejemplo para utilizarlas debidamente en este sentido (González, Lleixà \& Espuny, 2016; Siemens \& Weller, 2011). Otros estudios, como los de Domingo y Fuentes (2010), señalan al docente como el problema para dicha implementación. Por otro lado, también se han detectado ciertas reticencias en el uso de las redes sociales en el ámbito educativo al suponer una posible invasión de la privacidad del alumnado, el cual no siempre está dispuesto a perder dicha privacidad (Cruz, 2016).

Respecto a las modalidades de docencia, se distingue entre presencial, semipresencial y totalmente virtual $\mathrm{u}$ online. En el caso de las universidades presenciales, está claro que el uso de las redes sociales ha comportado un inevitable cambio de paradigma en el medio de aprendizaje en el que se desarrollaban los estudiantes. Así, se presenta una realidad distinta de la que había hace décadas, derivando en una forma diferente de comunicarse, buscar información y proceder en cuanto a las actuaciones y comportamientos del alumnado en las actividades cotidianas (Low, Polanin \& Espelage, 2013).

En esta línea, tal y como apuntaban Hortigüela y Pérez (2015), asumiendo la riqueza metodológica que otorga el hecho de compartir experiencias, ideas y proyectos con otros miembros de la comunidad educativa, la utilización de las redes sociales en un entorno presencial favorecía la creación de vínculos formativos que inciden en el tratamiento de una temática determinada. Para estos autores, la motivación era crucial, demostrando que lo importante no era el nivel que los alumnos adquieren en dicho uso, sino el modo en el que el docente orienta el proceso para obtener un mayor aprendizaje (Hortigüela \& Pérez, 2015).

Por otro lado, estudios como el de Martínez y Ferraz (2016) establecieron que los estudiantes presenciales valoraban positivamente el uso de las redes sociales como un instrumento académico para buscar solución de dudas con otros estudiantes, para realizar los trabajos de clase con otros compañeros, para buscar información, para consultar novedades surgidas en torno a la facultad y/o universidad donde el estudiante se encuentra matriculado, etc.

De este modo, las redes sociales en el contexto presencial se han constituido como un aliado para transmitir conocimientos, "divulgar información, compartir experiencias, y sobre todo analizar enfoques de la misma temática que tienen otros profesionales" (Hortigüela \& Pérez 2015, p. 100), mutando de plataformas con un fin social exclusivamente a una herramienta para compartir información.

En el caso de las universidades no presenciales, se ha acentuado la necesidad de interactuar a través de las redes sociales, pues en esta coyuntura no han funcionado como una cuestión complementaria en sus 
Tabla 1. Descripción de la muestra en función del género, edad y grado. Fuente: elaboración propia.

\begin{tabular}{|c|c|c|c|c|c|c|c|}
\hline & Número de participantes & Hombres & Mujeres & Edad & Infantil & Primaria & Doble titulación \\
\hline Presencial & 175 & $\begin{array}{l}24 \\
13.7 \% \\
\end{array}$ & $\begin{array}{l}151 \\
86.3 \% \\
\end{array}$ & $\begin{array}{l}M=20.99 \\
S D=3.137\end{array}$ & $\begin{array}{l}55 \\
31.4 \% \\
\end{array}$ & $\begin{array}{l}109 \\
62.3 \% \\
\end{array}$ & $\begin{array}{l}11 \\
6.3 \% \\
\end{array}$ \\
\hline Online & 165 & $\begin{array}{l}21 \\
12.7 \% \\
\end{array}$ & $\begin{array}{l}144 \\
87.3 \% \\
\end{array}$ & $\begin{array}{l}\mathrm{M}=33.49 \\
\mathrm{SD}=7.250\end{array}$ & $\begin{array}{l}71 \\
(43 \%) \\
\end{array}$ & $\begin{array}{l}89 \\
(53.9 \%) \\
\end{array}$ & $\begin{array}{l}5 \\
(3 \%) \\
\end{array}$ \\
\hline Total & 340 & $\begin{array}{l}45 \\
13.2 \%\end{array}$ & $\begin{array}{l}295 \\
86.8 \%\end{array}$ & $\begin{array}{l}M=27.06 \\
S D=8.345\end{array}$ & $\begin{array}{l}126 \\
37.1 \%\end{array}$ & $\begin{array}{l}198 \\
58.2 \%\end{array}$ & $\begin{array}{l}1 \\
4.7 \%\end{array}$ \\
\hline
\end{tabular}

relaciones profesionales y personales, sino que se han materializado como el medio que el alumnado tiene a su alcance para suplir la ausencia de contacto espacial existente entre ellos. Trabajos más relacionados con la sociología se posicionaron a favor de hacernos entender que el uso de las redes aporta determinados elementos humanos que incluían el ámbito afectivo y cubrían necesidades sociales, promoviendo un aprovechamiento del capital intelectual (Colás, Conde \& Martín, 2015; Al-Samarrie \& Saeed, 2018). A pesar de esto, se ha mostrado poca formación al alumnado en el uso y beneficio de las redes sociales en el ámbito académico virtual (Calderón-Garrido, León-Gómez \& Gil-Fernández, 2019).

Por tanto, se asume que en un entorno virtual son el instrumento que el discente posee para configurar verdaderas comunidades de aprendizaje (Tirado \& Martínez, 2010), pues, para que este hecho se produzca, debería tener un proyecto común, compartir herramientas y sobre todo fomentar la adquisición de la motivación necesaria para llegar a conseguir metas. En suma, las redes sociales permiten a este tipo de alumnos mantener conexiones (Rojas, 2014).

Así pues, la finalidad de este trabajo es conocer el uso que los maestros en su formación inicial hacen de las redes sociales genéricas, y en concreto cómo estas son empleadas con finalidades educativas, diferenciando dichos datos en función de la modalidad de enseñanza de cada institución. Esta investigación actualiza los estudios precedentes y, sobre todo, arroja nuevos datos sobre la modalidad educativa online, carente aún de estudios parecidos por su relativa novedad.

\section{Método}

Para la consecución de los objetivos propuestos se diseñó y validó un cuestionario ad hoc (disponible en https://reunir.unir.net/123456789/6695) de forma que se pudiese atender a las especificidades propias del alumnado de los grados de Maestro de Educación Infantil, Maestro de Educación Primaria y Doble Titulación de Infantil y Primaria, tanto en universidades presenciales como virtuales. Su administración fue online a través de la plataforma formsite, estableciéndose un periodo de tres semanas para su respuesta. El alumnado participante firmó un consentimiento libre, previo e informado implícito en el propio cuestionario, siendo conocedor de que podía abandonar el estudio en el momento que desease. El vaciado y posterior análisis de resultados se realizó con el software de análisis cuantitativo Statistical Package for the Social Science (SPSS) en su versión 21.0.
La muestra potencial se eligió aleatoriamente en diversas asignaturas de diferentes cursos, siendo un total de 1200 alumnos (510 de una universidad virtual y 690 de diversas universidades presenciales). Tras depurar datos, la muestra analizada fue de un total de 340 alumnos, 175 de diferentes universidades presenciales (Universidad de Barcelona, Universidad Camilo José Cela, Universidad Complutense de Madrid y Universidad de Sevilla) y 165 de los diferentes grados de Maestro de una universidad virtual (Universidad Internacional de La Rioja). En la tabla 1 se describe la muestra en función del género, la edad y la titulación.

Por lo que se refiere a la fiabilidad interna, el cuestionario ha mostrado un índice destacable (alfa de Cronbach .841; .839 en el caso de la universidad virtual y .845 en las universidades presenciales).

\section{Resultados}

Los resultados mostraron que las redes sociales preferidas por el alumnado eran WhatsApp, YouTube y Facebook. Por otro lado, se observó que WeChat, Tumblr y SoundCloud eran minoritarias y mostraban un uso casi anecdótico.

Respecto a su uso en educación, se observó que este era totalmente ocasional en la mayoría de las redes, con un poco más de asiduidad en YouTube, WhatsApp y Pinterest. En la tabla 2 se muestran los resultados obtenidos, tanto genéricos como en función de la presencialidad de los grados, los cuales serán desarrollados en el apartado de discusión y concusiones.

En función de las respuestas obtenidas, el análisis de cluster (prueba de esfericidad de Barlett $p<.001$ ) mostró tres grupos principales diferentes en función del uso educativo que realizaban de las diversas redes sociales: el referido al uso de SoundCloud, WeChat y Tumblr; un gran grupo de Facebook, Skype, Pinterest e Instagram; y un grupo de Twitter y WhatsApp. El uso de YouTube era transversal y común al resto de grupos.

En función del tipo de universidad (presencial $\mathrm{u}$ online), se mostraron diferencias estadísticamente significativas en tener una cuenta en Facebook $(Z=-1.969$; $p=.049)$, Instagram $(Z=-6.942 ; p<.001)$, Pinterest $(Z$ $=-3.406 ; p=.001)$, SoundCloud $(Z=-3.425 ; p=.001)$, Twitter $(Z=-3.781 ; p<.001)$, WhatsApp $(Z=-3.301 ; p$ $=.001)$ y YouTube $(Z=-3.162 ; p=.002)$.

En función del tipo de universidad (presencial u online), se observaron diferencias estadísticamente significativas en el uso de las redes sociales en educación en el caso de Facebook $(Z=-5.196 ; p<.001)$, Pinterest $(Z=-2.897 ; p<.004)$, Skype $(Z=-1.982 ; p=$ 
Tabla 2. Resultados obtenidos. Fuente: elaboración propia.

\begin{tabular}{|c|c|c|c|c|c|c|c|c|c|c|c|c|}
\hline & \multicolumn{4}{|l|}{ Tienen } & \multicolumn{8}{|c|}{ Usan en educación } \\
\hline & \multicolumn{2}{|l|}{ No } & \multicolumn{2}{|l|}{ Sí } & \multicolumn{2}{|l|}{ Nunca } & \multicolumn{2}{|l|}{ Alguna Vez } & \multicolumn{2}{|l|}{ A menudo } & \multicolumn{2}{|l|}{ Siempre } \\
\hline & Presencial & On line & Presencial & On line & Presencial & On line & Presencial & On line & Presencial & On line & Presencial & On line \\
\hline \multirow[t]{2}{*}{ Facebook } & $\begin{array}{l}55 \\
16.2 \% \\
\end{array}$ & & $\begin{array}{l}285 \\
83.8 \%\end{array}$ & & $\begin{array}{l}96 \\
33.7 \%\end{array}$ & & $\begin{array}{l}108 \\
37.9 \%\end{array}$ & & $\begin{array}{l}59 \\
20.7 \%\end{array}$ & & $\begin{array}{l}22 \\
7.7 \%\end{array}$ & \\
\hline & $\begin{array}{l}35 \\
20 \%\end{array}$ & $\begin{array}{l}20 \\
12.1 \%\end{array}$ & $\begin{array}{l}140 \\
80 \%\end{array}$ & $\begin{array}{l}145 \\
87.9 \%\end{array}$ & $\begin{array}{l}63 \\
45 \%\end{array}$ & $\begin{array}{l}33 \\
22.8 \%\end{array}$ & $\begin{array}{l}58 \\
41.4 \%\end{array}$ & $\begin{array}{l}50 \\
34,5 \%\end{array}$ & $\begin{array}{l}10 \\
7.1 \%\end{array}$ & $\begin{array}{l}49 \\
33.8 \%\end{array}$ & $\begin{array}{l}9 \\
6.4 \%\end{array}$ & $\begin{array}{l}13 \\
9 \%\end{array}$ \\
\hline \multirow[t]{2}{*}{ Instagram } & $\begin{array}{l}80 \\
23.5 \%\end{array}$ & & $\begin{array}{l}260 \\
76.5 \%\end{array}$ & & $\begin{array}{l}132 \\
50.8 \%\end{array}$ & & $\begin{array}{l}63 \\
24.2 \%\end{array}$ & & $\begin{array}{l}28 \\
10.8 \%\end{array}$ & & $\begin{array}{l}37 \\
14.2 \%\end{array}$ & \\
\hline & $\begin{array}{l}14 \\
8 \% \\
\end{array}$ & $\begin{array}{l}66 \\
40 \% \\
\end{array}$ & $\begin{array}{l}161 \\
92 \% \\
\end{array}$ & $\begin{array}{l}99 \\
60 \% \\
\end{array}$ & $\begin{array}{l}86 \\
53.4 \% \\
\end{array}$ & $\begin{array}{l}46 \\
46.5 \% \\
\end{array}$ & $\begin{array}{l}39 \\
24.2 \% \\
\end{array}$ & $\begin{array}{l}24 \\
24.2 \% \\
\end{array}$ & $\begin{array}{l}11 \\
6.8 \% \\
\end{array}$ & $\begin{array}{l}17 \\
17.2 \% \\
\end{array}$ & $\begin{array}{l}25 \\
15.5 \% \\
\end{array}$ & $\begin{array}{l}12 \\
12.1 \% \\
\end{array}$ \\
\hline \multirow[t]{2}{*}{ Pinterest } & $\begin{array}{l}139 \\
40.9 \% \\
\end{array}$ & & $\begin{array}{l}201 \\
59.1 \% \\
\end{array}$ & & $\begin{array}{l}25 \\
12.4 \% \\
\end{array}$ & & $\begin{array}{l}66 \\
32.8 \% \\
\end{array}$ & & $\begin{array}{l}75 \\
37.3 \% \\
\end{array}$ & & $\begin{array}{l}35 \\
17.4 \% \\
\end{array}$ & \\
\hline & $\begin{array}{l}87 \\
49.7 \%\end{array}$ & $\begin{array}{l}52 \\
31.5 \%\end{array}$ & $\begin{array}{l}88 \\
55.3 \%\end{array}$ & $\begin{array}{l}113 \\
68.5 \%\end{array}$ & $\begin{array}{l}17 \\
19.3 \%\end{array}$ & $\begin{array}{l}8 \\
7.1 \%\end{array}$ & $\begin{array}{l}30 \\
34.1 \%\end{array}$ & $\begin{array}{l}36 \\
31.9 \%\end{array}$ & $\begin{array}{l}32 \\
36.4 \%\end{array}$ & $\begin{array}{l}43 \\
38.1 \%\end{array}$ & $\begin{array}{l}9 \\
5.1 \%\end{array}$ & $\begin{array}{l}26 \\
23 \% \\
\end{array}$ \\
\hline \multirow[t]{2}{*}{ Skype } & $\begin{array}{l}99 \\
29.1 \% \\
\end{array}$ & & $\begin{array}{l}241 \\
70.9 \% \\
\end{array}$ & & $\begin{array}{l}107 \\
44.4 \% \\
\end{array}$ & & $\begin{array}{l}98 \\
40.7 \% \\
\end{array}$ & & $\begin{array}{l}25 \\
10.4 \% \\
\end{array}$ & & $\begin{array}{l}11 \\
4.6 \%\end{array}$ & \\
\hline & $\begin{array}{l}55 \\
31.4 \%\end{array}$ & $\begin{array}{l}44 \\
26.7 \%\end{array}$ & $\begin{array}{l}120 \\
68.6 \%\end{array}$ & $\begin{array}{l}121 \\
73.3 \%\end{array}$ & $\begin{array}{l}45 \\
37.5 \%\end{array}$ & $\begin{array}{l}62 \\
51.2 \%\end{array}$ & $\begin{array}{l}55 \\
45.8 \%\end{array}$ & $\begin{array}{l}43 \\
35.5 \%\end{array}$ & $\begin{array}{l}14 \\
11.7 \%\end{array}$ & $\begin{array}{l}11 \\
9.1 \%\end{array}$ & $\begin{array}{l}6 \\
5 \%\end{array}$ & $\begin{array}{l}5 \\
41 \%\end{array}$ \\
\hline \multirow[t]{2}{*}{ SoundCloud } & $\begin{array}{l}301 \\
88.5 \% \\
\end{array}$ & & $\begin{array}{l}39 \\
11.5 \% \\
\end{array}$ & & $\begin{array}{l}14 \\
35.9 \% \\
\end{array}$ & & $\begin{array}{l}16 \\
41 \% \\
\end{array}$ & & $\begin{array}{l}7 \\
17.9 \% \\
\end{array}$ & & $\begin{array}{l}2 \\
5.1 \% \\
\end{array}$ & \\
\hline & $\begin{array}{l}165 \\
94.3 \% \\
\end{array}$ & $\begin{array}{l}136 \\
82.4 \% \\
\end{array}$ & $\begin{array}{l}10 \\
5.7 \%\end{array}$ & $\begin{array}{l}29 \\
17.6 \% \\
\end{array}$ & $\begin{array}{l}8 \\
80 \% \text { o }\end{array}$ & $\begin{array}{l}6 \\
20.7 \% \\
\end{array}$ & $\begin{array}{l}2 \\
20 \% \\
\end{array}$ & $\begin{array}{l}14 \\
48.3 \%\end{array}$ & 0 & $\begin{array}{l}7 \\
24.1 \% \\
\end{array}$ & 0 & $\begin{array}{l}2 \\
6.9 \% \\
\end{array}$ \\
\hline \multirow[t]{2}{*}{ Tumblr } & $\begin{array}{l}307 \\
90.3 \% \\
\end{array}$ & & $\begin{array}{l}33 \\
9.7 \% \\
\end{array}$ & & $\begin{array}{l}20 \\
60.6 \% \\
\end{array}$ & & $\begin{array}{l}8 \\
24.2 \% \\
\end{array}$ & & $\begin{array}{l}3 \\
9.1 \% \\
\end{array}$ & & $\begin{array}{l}2 \\
6.1 \% \\
\end{array}$ & \\
\hline & $\begin{array}{l}153 \\
87.4 \% \\
\end{array}$ & $\begin{array}{l}154 \\
93.3 \% \\
\end{array}$ & $\begin{array}{l}22 \\
12.6 \% \\
\end{array}$ & $\begin{array}{l}11 \\
6.7 \% \\
\end{array}$ & $\begin{array}{l}12 \\
54.5 \% \\
\end{array}$ & $\begin{array}{l}8 \\
72.7 \% \\
\end{array}$ & $\begin{array}{l}6 \\
27.3 \% \\
\end{array}$ & $\begin{array}{l}2 \\
18.2 \% \\
\end{array}$ & $\begin{array}{l}3 \\
13.6 \% \\
\end{array}$ & 0 & $\begin{array}{l}1 \\
4.5 \%\end{array}$ & $\begin{array}{l}1 \\
9.1 \% \\
\end{array}$ \\
\hline \multirow[t]{2}{*}{ Twitter } & $\begin{array}{l}126 \\
37.1 \% \\
\end{array}$ & & $\begin{array}{l}214 \\
62.9 \% \\
\end{array}$ & & $\begin{array}{l}81 \\
37.9 \% \\
\end{array}$ & & $\begin{array}{l}84 \\
39.3 \% \\
\end{array}$ & & $\begin{array}{l}34 \\
15.9 \% \\
\end{array}$ & & $\begin{array}{l}15 \\
7 \% \\
\end{array}$ & \\
\hline & $\begin{array}{l}48 \\
27.4 \% \\
\end{array}$ & $\begin{array}{l}78 \\
47.3 \%\end{array}$ & $\begin{array}{l}127 \\
72.6 \% \\
\end{array}$ & $\begin{array}{l}87 \\
52.7 \% \\
\end{array}$ & $\begin{array}{l}48 \\
37.8 \% \\
\end{array}$ & $\begin{array}{l}33 \\
37.9 \% \\
\end{array}$ & $\begin{array}{l}53 \\
41.7 \% \\
\end{array}$ & $\begin{array}{l}31 \\
35.6 \%\end{array}$ & $\begin{array}{l}18 \\
14.2 \%\end{array}$ & $\begin{array}{l}16 \\
18.4 \%\end{array}$ & $\begin{array}{l}8 \\
6.3 \% \\
\end{array}$ & $\begin{array}{l}7 \\
8 \% \\
\end{array}$ \\
\hline \multirow[t]{2}{*}{ WhatsApp } & $\begin{array}{l}10 \\
2.9 \% \\
\end{array}$ & & $\begin{array}{l}330 \\
97.1 \%\end{array}$ & & $\begin{array}{l}41 \\
12.4 \%\end{array}$ & & $\begin{array}{l}100 \\
30.3 \%\end{array}$ & & $\begin{array}{l}123 \\
37.3 \%\end{array}$ & & $\begin{array}{l}66 \\
20 \% \\
\end{array}$ & \\
\hline & 0 & $\begin{array}{l}10 \\
6.1 \% \\
\end{array}$ & $\begin{array}{l}175 \\
100 \% \\
\end{array}$ & $\begin{array}{l}155 \\
93.9 \% \\
\end{array}$ & $\begin{array}{l}12 \\
6.9 \% \\
\end{array}$ & $\begin{array}{l}29 \\
18.7 \% \\
\end{array}$ & $\begin{array}{l}39 \\
22.3 \% \\
\end{array}$ & $\begin{array}{l}61 \\
39.4 \% \\
\end{array}$ & $\begin{array}{l}83 \\
47.4 \% \\
\end{array}$ & $\begin{array}{l}40 \\
25.8 \% \\
\end{array}$ & $\begin{array}{l}41 \\
23.4 \% \\
\end{array}$ & $\begin{array}{l}25 \\
16.1 \% \\
\end{array}$ \\
\hline \multirow[t]{2}{*}{ WeChat } & $\begin{array}{l}322 \\
94.7 \% \\
\end{array}$ & & $\begin{array}{l}18 \\
5.3 \% \\
\end{array}$ & & $\begin{array}{l}14 \\
77.8 \% \\
\end{array}$ & & $\begin{array}{l}3 \\
16.7 \% \\
\end{array}$ & & $\begin{array}{l}1 \\
5.6 \% \\
\end{array}$ & & 0 & \\
\hline & $\begin{array}{l}168 \\
96 \% \\
\end{array}$ & $\begin{array}{l}154 \\
93.3 \% \\
\end{array}$ & $\begin{array}{l}7 \\
4 \% \\
\end{array}$ & $\begin{array}{l}11 \\
6.7 \% \\
\end{array}$ & $\begin{array}{l}6 \\
85.7 \% \\
\end{array}$ & $\begin{array}{l}8 \\
72.7 \% \\
\end{array}$ & $\begin{array}{l}1 \\
14,3 \% \\
\end{array}$ & $\begin{array}{l}2 \\
18.2 \% \\
\end{array}$ & 0 & $\begin{array}{l}1 \\
9.1 \% \\
\end{array}$ & 0 & 0 \\
\hline \multirow[t]{2}{*}{ Youtube } & $\begin{array}{l}56 \\
16.5 \% \\
\end{array}$ & & $\begin{array}{l}284 \\
83.5 \% \\
\end{array}$ & & $\begin{array}{l}21 \\
7.4 \% \\
\end{array}$ & & $\begin{array}{l}87 \\
30.6 \% \\
\end{array}$ & & $\begin{array}{l}135 \\
47.5 \% \\
\end{array}$ & & $\begin{array}{l}41 \\
14.4 \% \\
\end{array}$ & \\
\hline & $\begin{array}{l}18 \\
10.3 \%\end{array}$ & $\begin{array}{l}38 \\
23 \%\end{array}$ & $\begin{array}{l}157 \\
89.7 \%\end{array}$ & $\begin{array}{l}127 \\
77 \%\end{array}$ & $\begin{array}{l}13 \\
8.3 \%\end{array}$ & $\begin{array}{l}8 \\
6.3 \%\end{array}$ & $\begin{array}{l}51 \\
32.5 \%\end{array}$ & $\begin{array}{l}36 \\
28.3 \%\end{array}$ & $\begin{array}{l}72 \\
45.9 \%\end{array}$ & $\begin{array}{l}63 \\
49.6 \%\end{array}$ & $\begin{array}{l}21 \\
13.4 \%\end{array}$ & $\begin{array}{l}20 \\
15.7 \%\end{array}$ \\
\hline
\end{tabular}

$.047)$, SoundCloud $(Z=-3.258 ; p=.001)$, WhatsApp $(Z$ $=-4.731 ; p<.001)$.

En función del género, los datos únicamente mostraron diferencias estadísticas en el uso con fines educativos de Facebook en las universidades presenciales $(Z=-2.760 ; p=.006)$, siendo las mujeres las más habituadas a su uso.

Respecto al grado, únicamente se observaron diferencias estadísticas en el uso de Facebook en Educación $\left(X_{(2)}^{2}=14.869 ; p=.001\right)$, siendo los alumnos de Educación Infantil de la universidad online los más proclives a su uso.

\section{Discusión}

Los datos analizados sobre el uso de las redes sociales obtenidos entre los maestros en su formación inicial mostraron que se decantan principalmente por WhatsApp (97.1\%), YouTube (83.5\%) y Facebook (83.8\%). Estos datos actualizan los estudios de Martínez y Ferraz (2016), quienes concluían que Facebook era la red social más conocida y usada entre los estudiantes universitarios. Esta discrepancia es el resultado de los rápidos cambios que la sociedad experimenta en el uso de las redes sociales, que en muchas ocasiones se deben a modas (Puente, Fernández, Sequeiros \& López, 2015) y a los movimientos en los diferentes grupos sociales (Franklin, 2019).

Respecto al género, se ha observado que no existen diferencias estadísticas. Tanto Cruz (2016) como Sanz, Alonso, Sáenz, Ponce y Valdemoros (2018) afirmaron que en el contexto español las mujeres, en la etapa de educación secundaria, eran más proclives al uso de las redes sociales que los hombres. Esta diferenciación entre ambas etapas apunta hacia una democratización de su uso a medida que los estudiantes se van haciendo mayores y llegan a la educación superior.

Tal como afirma Marcelino, "las poblaciones de distintas edades se enfocan en las redes más afines, pero, se movilizan de unas a otras si cambian sus intereses o si surgen espacios sociales que generen y/o suplan nuevas necesidades de comunicación en línea" (2015, p. 55). Por tanto, se puede afirmar que un conocimiento o uso de determinada red social en el día a día de una persona puede verse modificado en su etapa universitaria, adaptándose a las necesidades propias de dicha etapa. De tal forma, el estudiante se acercará hacia las redes sociales que le puedan ser útiles. Esto justifica la aparición de Pinterest como una de las redes sociales más usadas con fines educativos, porcen- 
tualmente hablando. Dicha red ya ha sido tratada en anteriores investigaciones, en las que se concluía que era una herramienta, además de útil, muy motivadora para el docente en su formación inicial (Amer \& Amer, 2018). También se observó un mayor uso educativo de WhatsApp, ya reflejada como la red social más común en estudiantes universitarios españoles por Fondevila, Marqués, Mir y Polo (2018).

Mención especial merece el caso de YouTube, que permite desarrollar distintas estrategias de aprendizaje entre el alumnado, tal como destacan Sábada y Rendueles (2016). Además de esto, en contextos educativos suele emplearse como repositorio de material audiovisual. Marchetti y Valente (2018) defendieron que su uso estaba vinculado al predominio de elementos visuales que promueven un aprendizaje más efectivo. Así pues, y tal y como apunta Ramírez-Ochoa, "los usuarios pueden elegir entre más de 20.000 vídeos creados por universidades como Stanford, UC Berkeley, UCLA y Yale" (2016, p. 540).

Sin embargo, y a pesar de ese discreto despunte de Pinterest, WhatsApp y YouTube, se observó un bajo uso de las redes sociales con fines educativos. Esto significa un desperdicio de las posibilidades e idoneidad de dichas redes en entornos formativos, obviando así el contacto interpersonal y espontáneo que las plataformas institucionales no ofrecen (Rojas, 2014).

En función de la tipología de universidad, llaman especialmente la atención las diferencias estadísticas mostradas en el caso de Facebook $(Z=-1.969 ; p=.049$; más habitual en la universidad online) e Instagram ( $Z$ $=-6.942 ; p<.001$; con mayor presencia en las universidades presenciales). Esto se debe principalmente a las diferentes horquillas de edad de su alumnado $(\mathrm{M}=33.4$ online y $\mathrm{M}=20.99$ presencial), ya que, tal como afirmaron Phua, Jin y Kim (2017), Instagram está más presente entre los menores de treinta años y Facebook entre los mayores de dicha edad.

Respecto al uso educativo, se observaron diferencias estadísticas en el uso de Facebook $(Z=-5.196 ; p<.001)$, siendo más habitual en la universidad online. Este resultado entronca con el empleo que estos alumnos hacen del perfil de la universidad en Facebook y, por ende, con la posibilidad que presenta este recurso como una estrategia de aprendizaje y promotor de socialización del alumnado (Martínez, 2014; Colás et al., 2015). A pesar de esto, y tal como se ha descrito, los resultados muestran, tanto en presencial como online, que su uso educativo no está demasiado generalizado entre el alumnado, por lo que sus beneficios, como el aprendizaje colaborativo (Al-Samierre \& Saed, 2018; Wamuyu, 2018), no se aprovechan en demasía.

En el caso del uso educativo de SoundCloud se observaron diferencias estadísticas $(Z=-3.258 ; p=$ .001), siendo esta más utilizada por los alumnos online que por los presenciales. Esto contrasta con su formato de notas de voz grabadas de contenido abierto, más propio de universidades presenciales (Nwosu, Monnery, Reid \& Chapman, 2017), ya que en la universidad online se decantan por archivos audiovisua- les que están disponibles como material de apoyo al aprendizaje.

También se observaron diferencias estadísticas en el caso de Skype $(Z=-1.982 ; p=.047)$, siendo más habitual entre los alumnos de las universidades presenciales. Esto puede ser debido a que el contacto entre el alumnado de la universidad online se suele generar en la propia plataforma en la que desarrollan las sesiones virtuales (adobeconnect), a pesar de que dicha plataforma es dirigida por el profesorado. En cualquier caso, su bajo uso en ambas modalidades sigue presentando un desaprovechamiento del aprendizaje colaborativo defendido para este recurso por Mnkandla y Minnar (2017).

En el caso de WhatsApp, a pesar de su abrumadora presencia en la muestra analizada, su uso educativo es mucho más bajo, mostrando diferencias estadísticas ( $Z$ $=-4.731 ; p<.001)$ y siendo más habitual entre el alumnado presencial. Esto posiblemente esté relacionado con el tipo de contacto que permite dicha tipología educativa, mientras que aquella online se limita a entornos virtuales.

Respecto a Twitter, se mostró cierta igualdad en su uso. Una revisión de los perfiles Twitter de cada institución analizada confirma un uso eminentemente educativo. A pesar de esto, se trata de un recurso poco aprovechado por el alumnado, lo que conlleva que desperdicien la posibilidad de intercambio continuo de ideas $y$, por ende, mayor enriquecimiento en su formación (Tur, Marín \& Carpenter, 2017; Köseoğlu \& Köksal, 2018).

Por lo que respecta al uso en función del grado, se ha reflejado que únicamente Facebook con finalidades educativas presenta diferencias estadísticas, en concreto en el caso del alumnado de Educación Infantil de la universidad online respecto al resto. Dichas diferencias están relacionadas con la edad, siendo este alumnado el mayor $(M=34.51 ; S D=7.03)$. Por tanto, no existen diferencias intrínsecas al grado ni a las competencias que en él se desarrollan en el uso de las redes sociales en educación.

En conclusión, se puede afirmar que el uso de las redes sociales está extendido entre todos los maestros en su formación inicial, mostrando una clara preferencia por las de mensajería instantánea, como es el caso de WhatsApp. A pesar de esto, su uso como herramienta educativa es más bien escaso, de forma que se desaprovechan las virtudes que cada red mencionada ofrece. En función de la universidad, hemos visto que el alumnado online está más sensibilizado que el presencial hacia dicho uso. Así pues, y a pesar de los esfuerzos realizados en pro de la tecnología educativa, aún queda mucho camino por recorrer en lo que a su aplicabilidad a través de las redes sociales se refiere.

\section{Apoyos}

Este trabajo ha sido financiado por el proyecto "Evaluación de la Competencia Digital Docente en la formación inicial de docentes del área de Ciencias Socia- 
les y sus Didácticas Específicas", desarrollado dentro del grupo de investigación EDUC-07 INCISO (Didáctica de las Ciencias Sociales e Innovación Pedagógica en la Sociedad Digital, de la Universidad Internacional de La Rioja, Facultad de Educación).

\section{Declaración de divulgación de los autores:}

No existen intereses en conflicto.

\section{Referencias}

Altuzarra, A., Gálvez, C. \& Gónzalez, A. (2018). Explorando el potencial de los dispositivos electrónicos y de las redes sociales en el proceso enseñanza-aprendizaje de los universitarios. Edutec. Revista Electrónica de Tecnología Educativa, (64), 18-40. https://doi. org/10.21556/edutec.2018.64.1031

Al-Samarrie, H. \& Saeed, N. (2018). A systematic review of cloud computing tools for collaborative learning: Opportunities and challenges to the blended-learning environment. Computers \& Education, 124, 7791. https://doi.org/10.1016/j.compedu.2018.05.016

Amer, B. \& Amer, T.S. (2018). Use of Pinterest to Promote Teacher-Student Relationships in a Higher Education Computer Information Systems Course. Journal of the Academy of Business Education, 19, 132-141.

Calderón, D., Forés, A. \& Gustems, J. (2015). Aproximación a las fortalezas de carácter en los estudiantes de Educación Social. Perfil de una muestra. REIRE, 9(2), 48-64. https://doi.org/10.1344/reire2016.9.2924

Calderón-Garrido, D., León-Gómez, A. \& Gil-Fernández, R. (2019). El uso de las redes sociales entre los estudiantes de grado de maestro en un entorno exclusivamente online. Vivat Academia. Revista de Comunicación, 147, 23-40. http://doi.org/10.15178/ va.2019.147.23-40.

Callaghan, N. \& Bower, M. (2012). Learning Through Social Networking Sites. The Critical Role of the Teacher. Educational Media International, 49(1), 1-17. https://doi.org/10.1080/09523987.2012.662621

Colás, P., Conde, J. \& Martín, A. (2015). Las redes sociales en la enseñanza universitaria: Aprovechamiento didáctico del capital social e intelectual. Revista Interuniversitaria de Formación del Profesorado, 83, 105-116.

Cruz, I. M. (2016). Percepciones en el uso de las redes sociales y su aplicación en la enseñanza de las matemáticas. Píxel-Bit, 48, 165-186. http://dx.doi. org/10.12795/pixelbit.2016.i48.11

Domingo, M. \& Fuentes, M. (2010). Innovación educativa: experimentar con las TIC y reflexionar sobre su uso. Pixel-Bit, 36, 171.180. https://www.redalyc. org/pdf/368/36815128013.pdf

Fondevila, J. F., Olmo, J. del \& Sierra, J. (2012). Búsqueda de información y redes sociales: el caso de la universidad. Vivat Academia, XIV (Especial), 497-511.

Fondevila, J. F., Marqués, J., Mir, P. \& Polo, M. (2018). Usos del WhatsApp en el estudiante universitario español. Pros y contras. Revista Latina de Comunicación Social, (73), 308-324. https://doi.org/10.4185/ RLCS-2019-1332

Franklin, T. (2019). The State of Social Media. Encontent, 42(1), 25-27.

García, A. \& Martín, A. (2013). Aplicando el EEES en la Universidad española: un estudio de caso sobre la utilización de metodologías 2.0 en las nuevas titulaciones de grado. Historia y comunicación social, 18(1), 603-613. https://doi.org/10.5209/rev_HICS.2013. v18.43992

González, C. \& Muñoz, L. (2016). Redes Sociales su impacto en la Educación Superior: Caso de estudio. Universidad Tecnológica de Panamá. Campus Virtuales, 5(1), 84-90.

González, J., Lleixà, M. \& Espuny, C. (2016). Las redes sociales y la educación superior: las actitudes de los estudiantes universitarios hacia el uso educativo de las redes sociales, de nuevo a examen. Education in the knowledge Society (EKS), 17(2), 21-38. http://dx.doi.org/10.14201/eks20161722138

Haro, J. J. de (2011). Redes Sociales para la educación, Madrid: Anaya.

Hortigüela, D. \& Pérez, Á. (2015). Uso de las redes sociales como elemento formativo en el aula: Análisis de la motivación del alumnado universitario, Icono 14, volumen (13), pp. 95-115. https://doi.org/10.7195/ ri14.v13i2.788

Köseoğlu, P. \& Köksal, M. S. (2017). An Analysis of Prospective Teachers' Perceptions Concerning the Concept of "Social Media" through Metaphors. Eurasia Journal of Mathematics, Science and Technology Education, 14(1), 45-52. https://doi.org/10.12973/ ejmste/79325

López-Fernández, Ma C. (2016). Redes Sociales en la Educación Superior: Implementación educativa y pedagógica en los procesos de enseñanza y aprendizaje. En R. Roig-Vila (Ed.), Tecnología, innovación e investigación en los procesos de enseñanza-aprendizaje, pp. 785-792. Barcelona: Octaedro.

López-Frías, C. (2014). La interrelación entre las redes sociales específicas de la comunicación científica y las redes sociales de uso general. Vivat Academia, 127, $103-116$. http://dx.doi.org/10.15178/ va.2014.127.103-116

Low, S., Polanin, J. \& Espelage, D. (2013). The Role os Social Networks in Physical and Relational Aggression among Young Adolescents. Journal of Youth and Adolescence, 42(7), 1078-1089. http://dx.doi.org/10.1007/ s10964-013-9933-5

Marcelino, G. V. (2015). Migración de los jóvenes españoles en redes sociales, de Tuenti a Facebook y de Facebook a Instagram. La segunda migración, Icono 14, 13(2), 48-72. https://doi.org/10.7195/ri14. v13i2.821

Marchetti, E. \& Valente, A. (2018). Interactivity and multimodality in language learning: the untapped potential of audiobooks. Universal Access in the Information Society, 17(2), 257-274. https://doi. org/10.1007/s10209-017-0549-5 
Marina, J. A., Pellicer, C. \& Manos, J. (2015). Libro blanco de la profesión docente y su entorno escolar. Ministerio de Educación, Cultura y Deporte: Madrid.

Martínez, O. (2014). Modelos de enseñanza y aprendizaje en la Universidad: Redes sociales y aplicaciones móviles para la generación de trabajos en grupo. En J. F. Durán y S. Durán (Eds.), La era de las TIC en la nueva docencia, 267-200. Madrid: McGraw Hill Education.

Martínez, M. C. \& Ferraz, E. (2016). Uso de las redes sociales por los alumnos universitarios de educación: un estudio de caso de la península ibérica, Tendencias Pedagógicas, 28, 33-44.

Mnkandla, E. \& Minnaar, A. (2017). The Use of Social Media in E-Learning: A Metasynthesis. The International Review of Research in Open and Distributed Learning, 18(5), 227-248. http://dx.doi.org/10.19173/ irrodl.v18i5.3014.

Nwosu, A. C., Monnery, D., Reid, V. L. \& Chapman, L. (2017). Use of podcast technology to facilitate education, communication and dissemination in palliative care: the development of the AmiPal podcast. BMJ supportive \& palliative care, 7(2), 212-217. http:// dx.doi.org/10.1136/bmjspcare-2016-001140

Orden ECD/65/2015, de 21 de enero, por la que se describen las relaciones entre las competencias, los contenidos y los criterios de evaluación de la educación primaria, la educación secundaria obligatoria y el bachillerato. $B O E \mathrm{n}^{\circ} 25$, de 29 de enero de 2015, 6986-7003.

Phua, J., Jin, S. V. \& Kim, J. J. (2017). Gratifications of using Facebook, Twitter, Instagram, or Snapchat to follow brands: The moderating effect of social comparison, trust, tie strength, and network homophily on brand identification, brand engagement, brand commitment, and membershipintention. Telematics and Informatics, 34(1), 412-424. https://doi. org/10.1016/j.tele.2016.06.004

Puente, H., Fernández, M., Sequeiros, S. \& López, M. (2015). Los estudios sobre jóvenes y TICs en España. Los estudios sobre la juventud en España: Pasado, presente, futuro, 110, 155-172. http://www.injuve.es/ sites/default/files/2017/46/publicaciones/revista110_ completa.pdf\#page $=156$

Recomendación 2006/962/CE del Parlamento Europeo y del Consejo, de 18 de diciembre de 2006, sobre las competencias clave para el aprendizaje permanente. Diario Oficial de la Unión Europea L 394.
Rojas, C. A. (2014). Uso de Facebook en ámbitos educativos universitarios: consideraciones y recomendaciones. Apertura, 6(2), 1-12.

Sádaba, I. \& Rendueles, C. (2016). Metodologías de análisis del espacio audiovisual online : Entre la innovación y la ansiedad de la novedad. Empiria. Revista de metodología de Ciencias Sociales, O(35), 105124. https://doi.org/10.5944/empiria.35.2016.17170 Sánchez, Mª M., Prendes, Ma Paz \& Serrano J. L. (2011). Modelos de interacción de los adolescentes en contextos presenciales y virtuales. Edutec-e, revista electrónica de tecnología educativa, 35.

Sanz, E., Alonso, R., Saénz, M., Ponce, A. \& Valdemoros, M. (2018). Ocio, Redes Sociales y estudiantes españoles. Educación XX1, 21(2). https://doi.org/10.5944/ educxx1.19538

Serrat, N. (2015). Metodologías participativas y Facebook en el ámbito universitario. Innoeduca. Journal of Technology and Educational Innovation, 1(1), 25-32. http:// dx.doi.org/10.20548/innoeduca.2015.v1i1.104

Siemens, G. \& Weller, M. (2011). La enseñanza superior y las promesas y los peligros de las redes sociales. En G. Siemens \& M. Weller (Coords.), El impacto de las redes sociales en la enseñanza y el aprendizaje. Revista de Universidad y Sociedad del Conocimiento (RUSC), 8(1), 157-163. Recuperado de http://rusc.uoc.edu/ojs/index.php/rusc/article/view/v8n1-siemens-weller/ v8n1-siemens-weller

Sloep, P. \& Berlanga, A. (2011). Redes de aprendizaje, aprendizaje en red. Comunicar, 19(37), 55-64. http:// dx.doi.org/10.3916/C37-2011-02-05

Tirado, R. \& Martínez, J. M. (2010). Creando comunidades virtuales de aprendizaje: análisis del progreso de las interacciones. En C. Coll \& L. Falsafi (Eds.), Identidad y Educación [Monográfico], Revista de educación, 353, 297-328.

Túnez, M. \& Sixto, J. (2012). Las redes sociales como entorno docente: análisis del uso de Facebook en la docencia universitaria. Pixel-Bit, Revista de Medios y Educación, 41, 77-92.

Tur, G., Marín, V. \& Carpenter, J. (2017). Uso de Twitter en Educación Superior en España y Estados Unidos. Comunicar, 51(25), 19-28. http://dx.doi. org/10.3916/C51-2017-02

Wamuyu, P. K. (2018). Leveraging Web 2.0 technologies to foster collective civic environmental initiatives among low-income urban communities. Computers in Human Behavior, 85, 1-14. https://doi.org/10.1016/j. chb.2018.03.029. 